INPLASY

PROTOCOL

To cite: Fukuta et al. Sodiumglucose cotransporter 2 inhibitors in heart failure with preserved ejection fraction: a protocol for meta-analysis. Inplasy protocol 2021120033. doi:

10.37766/inplasy2021.12.0033

Received: 06 December 2021

Published: 06 December 2021

Corresponding author: Hidekatsu Fukuta

fukuta-h@med.nagoya-cu.ac.jp

Author Affiliation:

Nagoya City University Graduate School of Medical Sciences.

Support: The faculty research expenses.

Review Stage at time of this submission: Preliminary searches.

Conflicts of interest:

None declared.

\section{Sodium-glucose cotransporter 2 inhibitors in heart failure with preserved ejection fraction: a protocol for meta-analysis}

\author{
Fukuta, $\mathrm{H}^{1}$; Hagiwara, $\mathrm{H}^{2}$; Kamiya, $\mathrm{T}^{3}$.
}

Review question / Objective: Nearly half of patients with heart failure (HF) have preserved ejection fraction (EF) and the mortality and morbidity of patients with HF with preserved EF (HFpEF) are high. Patients with HFpEF are often elderly and their primary chronic symptom is severe exercise intolerance that results in a reduced quality of life. Thus, improvement of exercise capacity presents another important clinical outcome in HFpEF patients. Recent randomized controlled trials (RCTs) and meta-analyses of RCTs reported that sodium-glucose cotransporter 2 (SGLT-2) inhibitors improved cardiovascular outcomes in patients with HF with reduced EF. Although the effects of SGLT-2 inhibitors in HFpEF patients have been examined in multiple RCTs, the results are inconsistent due partly to limited power. The purpose of this meta-analysis is to evaluate the efficacy and safety of SGLT-2 inhibitors in HFpEF patients.

Condition being studied: Stable patients with heart failure with preserved ejection fraction.

INPLASY registration number: This protocol was registered with the International Platform of Registered Systematic Review and Meta-Analysis Protocols (INPLASY) on 06 December 2021 and was last updated on 06 December 2021 (registration number INPLASY2021120033).

\section{INTRODUCTION}

Review question / Objective: Nearly half of patients with heart failure (HF) have preserved ejection fraction (EF) and the mortality and morbidity of patients with HF with preserved EF (HFpEF) are high. Patients with HFpEF are often elderly and their primary chronic symptom is severe exercise intolerance that results in a reduced quality of life. Thus, improvement of exercise capacity presents another important clinical outcome in HFpEF patients. Recent randomized controlled trials (RCTs) and meta-analyses of RCTs reported that sodium-glucose 
cotransporter 2 (SGLT-2) inhibitors improved cardiovascular outcomes in patients with HF with reduced EF. Although the effects of SGLT-2 inhibitors in HFpEF patients have been examined in multiple RCTs, the results are inconsistent due partly to limited power. The purpose of this meta-analysis is to evaluate the efficacy and safety of SGLT-2 inhibitors in HFpEF patients.

Condition being studied: Stable patients with heart failure with preserved ejection fraction.

\section{METHODS}

Search strategy: The electronic databases for literature search will include PubMed, Scopus, Cochrane Library, and Web of Science electronic databases. For search of the eligible studies, the following key words and Medical Subject Heading were used: diastolic heart failure, heart failure with normal (preserved) ejection fraction, randomized, sodi u m-g l u cose cotransporter 2 inhibitor(s). Only articles published in the English language will be included.

Participant or population: Patients with heart failure with preserved ejection fraction (HFpEF).

Intervention: Sodium-glucose cotransporter 2 inhibitors.

Comparator: Patients who are on standard medical therapy or placebo control group.

Study designs to be included: Randomized controlled trials (RCTs).

Eligibility criteria: Studies will be considered eligible if they; (1) included HFpEF patients; (2) were RCT; (3) used SGLT-2 inhibitors; (4) compared with usual medical therapy or placebo control group; and (5) assessed heart failure severity.

Information sources: PubMed, Scopus, Cochrane Library, and Web of Science electronic databases.
Main outcome(s): The primary outcome will be heart failure severity. In the measures of heart failure severity, plasma B-type natriuretic peptide levels and exercise capacity assessed as 6 minute-walk distance will be extracted.

Additional outcome(s): The secondary outcome will be health-related quality of life assessed as The Kansas City Cardiomyopathy Questionnaire. The safety outcomes of interest will be all-cause death, heart failure hospitalization, hypotension, acute renal failure, diabetic ketoacidosis, and urinary tract infection.

Quality assessment / Risk of bias analysis: The Cochrane Risk of Bias tool will be used to assess quality of RCTs included. The quality of evidence for the outcomes will be evaluated by use of the Grading of Recommendations Assessment, Development and Evaluation (GRADE) system. The quality of evidence will be evaluated across the domains of risk of bias, consistency, directness, precision, and publication bias.

Strategy of data synthesis: For continuous outcomes, the effect size for the intervention will be calculated by the difference between the means of the intervention and control groups at the end of the intervention. When available, the mean difference with corresponding standard deviation (SD), standard error of the mean (SEM) or confidence interval (CI) will be directly extracted from the article. When mean values (SD) at baseline and at the end of intervention were reported but the SD of the change or the correlation of the pre and post measurements is not available, the correlation will be conservatively set at 0.5 as previously reported. When the outcome is reported as median (range and/or interquartile range), the mean and SD will be estimated as previously reported. If the outcome is measured on the same scale, the weighted mean difference (WMD) and $95 \% \mathrm{Cl}$ will be calculated. Otherwise, the standardized mean difference (SMD) and $95 \% \mathrm{CI}$ will be calculated. For each outcome, heterogeneity will be assessed using the 
Cochran's $Q$ and 12 statistic; for the Cochran's $Q$ and 12 statistic, a $p$ value of $50 \%$, will be considered significant, respectively. When there is significant heterogeneity, the data will be pooled using a random-effects model, otherwise a fixedeffects model will be used. For categorical outcomes, the pooled estimate of odds ratio (OR) and $95 \% \mathrm{Cl}$ will be calculated with a fixed-effects model. When there is significant heterogeneity, the data will be pooled using a random-effects model. Event numbers will be either directly extracted or calculated.

Subgroup analysis: Meta-analysis will be performed separately for RCTs that included patients with $E F \geq 50 \%$ and those that included patients with $E F \geq 40 \%$.

Sensitivity analysis: Meta-regression will be used to determine whether the effect of SGLT-2 inhibitors will be confounded by baseline clinical characteristics.

Country(ies) involved: Japan.

Keywords: sodium-glucose cotransporter 2 inhibitors, heart failure, meta-analysis.

Contributions of each author:

Author 1 - Hidekatsu Fukuta.

Email: fukuta-h@med.nagoya-cu.ac.jp

Author 2 - Hiromi Hagiwara.

Email: crhiroha@med.nagoya-cu.ac.jp

Author 3 - Takeshi Kamiya.

Email: Takeshi Kamiya 